\title{
Vergleich unterschiedlicher Materialmodelle an einer
}

\section{Aushubsimulation}

Die Wahl eines passenden Materialmodells, welches in der Lage ist das Bodenverhalten bei FE-Berechnungen realistisch dazustellen, hat einen maßgeblichen Einfluss auf die dabei erhaltenen Berechnungsergebnisse. Anhand von Vergleichsrechnungen mit mehreren Materialmodellen sollen die Gemeinsamkeiten und Unterschiede bei den Ergebnissen verdeutlicht werden. Hierfür wird ein Baugrubenaushub simuliert und sowohl die auftretenden Verformungen, als auch die mittels Parameterreduktion ermittelte Standsicherheit betrachtet. Dabei wird neben den bekannteren Modellen Hardening Soil und der Hypoplastizität, die Barodesie als Modell für FE-Berechnungen vorgestellt. Während sich bei allen Modellen vergleichbare Verformungen einstellen, werden bei der berechneten Standsicherheit deutliche Unterschiede festgestellt.

Keywords: Barodesie, Hypoplastizität, Hardening Soil, Finite Elemente, Standsicherheit

Comparison of different material models by simulation of an excavation: The choice of a suitable material model, which is able to represent the soil behaviour in FE-simulations realistically, has a significant influence on the calculation results obtained. With the help of comparative calculations using several material models, the similarities and differences in the results will be clarified. For this purpose, an excavation is simulated and both the occurring deformations and the stability determined by parameter reduction are considered. Besides the more known models Hardening Soil and Hypoplasticity, Barodesy is presented as model for FEcalculations. While all models show comparable deformations, the calculated stability shows clear differences.

Keywords: Barodesy, Hypoplasticity, Hardening Soil, Finite Elements, stability

\section{$1 \quad$ Einleitung}

Die Methode der Finiten Elemente ist mittlerweile ein Standardwerkzeug zur Untersuchung geotechnischer Problemstellungen. Die Wahl eines passenden Materialmodells ist hierbei eine unerlässliche Aufgabe, da die Qualität der Ergebnisse stark vom gewählten Modell und dessen Kalibrierung abhängt. Während in der Forschung eine stetige Entwicklung der Materialmodellierung stattfindet, wird in der Praxis oftmals auf alte, einfache Modelle zurückgegriffen [1].

Es gibt bereits eine Vielzahl an Untersuchungen über den Einfluss unterschiedlicher Materialmodelle auf die Verformungsprognosen bei Finite Elemente Berechnungen (z.B. [2], [3], [4], [5]), welche die Unterschiede der Ergebnisse bei der Verwendung verschiedener Materialmodelle aufzeigen. Beispielsweise werden von Schweiger et. al. [2] mehrere elastoplastische Materialmodelle zur Verformungsprognose bei Baugruben angewandt. Dabei wird unter anderem die Notwendigkeit aufgezeigt, für undrainierte Verhältnisse höherwertige Materialmodelle zu verwenden, welche in der Lage sind die Spannungspfade realistisch abzubilden. In [5]

This is the author manuscript accepted for publication and has undergone full peer review but has not been through the copyediting, typesetting, pagination and proofreading process, which may lead to differences between this version and the Version of Record. Please cite this article as doi:

$10.1002 /$ gete. 201800016

Submitted: $\quad$ 18-Sep-2018

Revised: 20-Dec-2018

Accepted: 21-Dec-2018 
wird ein Baugrubenverbau mit unterschiedlichen Materialmodellen, unter anderem dem hypoplastischen Modell für Ton [6] sowie dem Modified-Cam-Clay Modell berechnet und mit Messwerten verglichen.

Eine starke Abhängigkeit der prognostizierten Verformungen des Baugrunds von der Wahl des Materialmodells wird auch in den Empfehlungen des Arbeitskreises Numerik in der Geotechnik [4] - kurz EANG - propagiert. Dort kommt man zu dem Schluss, dass einfache elasto-plastische Materialmodelle, beispielsweise nach Mohr-Coulomb, oftmals unrealistische Verformungen ergeben können.

Mittels Reduktion der Materialparameter lässt sich bei der FE-Methode die Standsicherheit abschätzen. Die allgemeine Eignung und Anwendbarkeit dieses Verfahrens wird u.a. von Tschuchnigg et. al. [7] untersucht. In der EANG wird festgestellt, dass die mittels Parameterreduktion ermittelte Standsicherheit eines Bauwerks nur gering von der Wahl des Materialmodells abhängt. In [8] wird diese Aussage kritisch betrachtet und hinterfragt, da diese im Allgemeinen nur schwer zu bestätigen ist. Im Gegensatz zur Verformungsprognose findet man jedoch nur kaum Vergleiche verschiedener Materialmodelle bei der Standsicherheitsberechnung und bei diesen liegt den Modellen für gewöhnlich das gleiche Versagenskriterium zu Grunde.

Es ist allgemein die Meinung verbreitet, dass höherwertige Materialmodelle zu viele Materialparameter benötigen, deren Bestimmung zu komplex und mit konventionellen Laborversuchen nicht möglich ist (z.B. [1], [9]). Dass eine große Anzahl an Materialparametern für eine realistische Abbildung des Bodenverhaltens jedoch nicht zwingend erforderlich ist, zeigen die in diesem Aufsatz verwendeten Materialmodelle. Während für eine Berechnung mit dem in der Praxis weit verbreiteten Hardening Soil Modell [10] acht Materialparameter benötigt werden, sind dies für die Hypoplastizität von Mašín [11] nur fünf und für die Barodesie für Ton [12] nur vier Materialparameter, welche alle anhand konventioneller Laborversuche zu bestimmen sind. Zählt man die zu Berechnungsbeginn festzulegende Eingangsporenzahl ebenfalls zu den Materialparametern, kommt hierfür noch eine Größe hinzu.

In dieser Arbeit wird die Barodesie (z.B. [12], [13], [14]) als Materialmodell für Finite Elemente-Berechnungen vorgestellt. Hierfür wird der Aushub einer, durch eine einfach gestützte Spundwand gesicherten, Baugrube in weichem Boden simuliert. Dabei soll der Einfluss des Materialmodells auf die Berechnungsergebnisse betrachtet werden. Hierfür werden zum einen die Unterschiede in den durch den Aushub auftretenden Verformungen und zum anderen die mittels Parameterreduktion auftretenden Unterschiede in der Standsicherheit verglichen.

Für die Auswertung der Berechnungsergebnisse wird der Spannungstensor mit $\boldsymbol{\sigma}^{\prime}$ bezeichnet. Dabei sind die Spannungen in bodenmechanischer Konvention mit positiven Druckspannungen angegeben. Mit $\sigma_{1}^{\prime}>\sigma_{2}^{\prime}>\sigma_{3}^{\prime}$ werden die effektiven Hauptspannungen bezeichnet.

\section{Materialmodelle}

Neben der Barodesie (BARO) werden für die Berechnungen ein elastoplastisches Materialmodell, Hardening Soil (HS), sowie ein hypoplastisches Materialmodell (HYPO) verwendet. Die Problemstellung gestützter Baugruben kann nach [1] bereits als niederzyklisches Randwertproblem betrachtet werden, womit auch die Anwendung von Modellen für zyklische Belastung denkbar wäre. Hardening Soil ist in Plaxis bereits standardmäßig implementiert. Die beiden anderen Modelle werden für die Berechnungen in Plaxis mittels spezieller Subroutinen, sogenannter user defined soil models (UDSM), implementiert, welche auf der Plattform soilmodels.com frei [15] verfügbar sind.

\subsection{Barodesie}

Die Barodesie (z.B. [12], [13]) ist ein Materialmodell, das sich von elasto-plastischen Modellen dadurch unterscheidet, dass keine Unterteilung der Verzerrungen in einen elastischen und plastischen Anteil getroffen wird. Im Modell wird die Spannungsrate als eine tensorielle Funktion der aktuellen effektiven Spannung, der Verformungsrate und der Porenzahl e formuliert. Die Barodesie hat damit Ähnlichkeiten mit der Hypoplastizität und beinhaltet gängige Konzepte der Critical State Soil Mechanics, vgl. [16]. Die hier verwendete Version der Barodesie für Ton [12] beinhaltet vier Materialparameter $\varphi_{c}^{\prime}, N, \lambda^{*}$ und $\kappa^{*}$, welche unabhängig vom Spannungszustand und der Lagerungsdichte des Bodens sind (Bild 1). Die FE-Implementierung des Materialmodells basiert auf Ideen von [17] und konnte bereits für mehrere Untersuchungen (z.B. [18], [19], [20]) erfolgreich angewandt werden. 


\subsection{Hardening Soil}

Bei Hardening Soil ([21], [10]) handelt es sich um ein elasto-plastisches Materialmodell mit Verfestigung und spannungsabhängiger Steifigkeit. In einem isotrop konsolidierten Triaxialversuch ist die deviatorische Fließfläche zunächst identisch mit der hydrostatischen Achse. Im Zuge des Abscherens wächst sie an, bis sie mit der Grenzbedingung nach Mohr-Coulomb zusammenfällt (Scherverfestigung). Bei einem $K_{0}$-konsolidierten Triaxialversuch ist die deviatorische Fließfläche bereits mobilisiert. Während der Scherverfestigung nimmt die Schubsteifigkeit stetig ab. Bei einer Entlastung und anschließender Wiederbelastung verhält sich das Material elastisch, solange man dabei nicht wieder auf eine Fließfläche trifft. Es werden für eine Berechnung acht Materialparameter benötigt, deren Bedeutung in [21] erläutert wird.

\subsection{Hypoplastizität}

Für die Berechnung wird die Hypoplastizität in der Version von Mašín [11] verwendet, welche speziell für feinkörnige Böden entwickelt wurde. Wie auch bei der Barodesie ist bei der Hypoplastizität die Spannungsrate in einer einzigen tensoriellen Gleichung gegeben. Durch die Berücksichtigung der Porenzahl kann der Einfluss der Lagerungsdichte berücksichtigt werden. Das Modell benötigt fünf Materialparameter von denen die vier Größen $\varphi_{c}^{\prime}, N, \lambda^{*}$ und $\kappa^{*}$, jenen der Barodesie entsprechen. Weiters wird der Parameter $v$ benötigt, welcher das Verhältnis von Kompressions- zu Schubsteifigkeit steuert. Für weitere Informationen über die Formulierung und Kalibrierung des Modells wird auf [11] verwiesen.

\section{$3 \quad$ FE-Berechnung}

Es wird die Berechnung eines Baugrubenaushubs durchgeführt. Die in Bild 2 dargestellte Geometrie sowie die Randbedingungen des Modells werden in Anlehnung an die Beispiele des Beiblatts 1 der EANG [4], bzw. [2] gewählt. Es handelt sich hierbei um eine Baugrube in einem weichen Boden, welcher mit Klei bezeichnet wird. Das Grundwasser in der Baugrube wird gemeinsam mit dem Boden ausgehoben. Unter der Annahme einer sehr geringen Durchlässigkeit des Bodens, stellt sich während des Aushubs praktisch keine Grundwasserströmung ein.

\subsection{Ermittlung der Materialparameter}

In [4] sind Materialparameter für verschiedene elastoplastische Materialmodelle, u.a. Hardening Soil, vorgegeben. Dort wird der Boden als normalkonsolidierter Klei mit einer Kohäsion von $15 \mathrm{kPa}$ angegeben. Nach dem Konzept von Krey-Tiedemann ([22], [23]) existiert eine sogenannte Kohäsion nur bei stark überkonsoliderten Böden. Diese kann mit Hilfe von Konzepten aus der Critical State Soil Mechanics wie folgt erläutert werden: Die sog. Critical State Line (CSL) im e-p' Diagramm trennt lockeren bzw. normal bis leicht überkonsolidierten $\left(e>e_{c}\right.$ und OCR $<2$ ) von dichtem bzw. stark überkonsolidiertem $\left(e<e_{c}\right.$ und OCR $\left.>2\right)$ Boden, vgl. Bild 3 (a). Dichter Boden zeigt im Spannungs-Stauchungs Diagramm einen Peak mit anschließender Entfestigung, welche bei lockerem Boden fehlt, vgl. Bild 3 (b). Peakzustände von stark überkonsolidiertem/dichtem Boden (rote Linie) liegen über der Critical State Line (CSL) im p'-q Diagramm, die für normal und leicht überkonsolidierte/lockere Böden maßgebend ist, vgl. Bild 3 (c).

Aus einer bestimmten Anfangsporenzahl resultiert also eine Festigkeitsumhüllende, die sich (für dichte Böden) zum Ursprung hin krümmt. Dies bestätigen auch Experimente von [24] an gesättigten, aufbereiteten Dresdner Ton Proben, in denen selbst bei stark überkonsolidiertem Ton die Scherfestigkeit für $\mathrm{p}^{\prime}=0 \mathrm{kPa}$ verschwindet.

Diese Festigkeitsumhüllende der Peakzustände wird nach Krey-Tiedemann mit der roten Gerade in Bild 4 (a) approximiert. Die Neigung der roten Linie ist $\mathrm{M}<\mathrm{M}_{\mathrm{c}}$ und aus $\mathrm{M}=\frac{6 \sin \varphi^{\prime}}{3-\sin \varphi^{\prime}}$ kann ein Reibungswinkel für ein Mohr-Coulomb Modell ermittelt werden. Damit ergibt sich für stark überkonsolidierte Böden eine Approximation an die Peakzustände mit $\mathrm{c}^{\prime}>0$ und $\varphi^{\prime}<\varphi_{\mathrm{c}}^{\prime}$. Aus dem Ordinatenabschnitt $\mathrm{q}_{0}$ folgt die Kohäsion $\mathrm{c}^{\prime}=\frac{\mathrm{q}_{0}}{\mathrm{M}} \cdot \tan \varphi^{\prime}$. Für normal bis leicht überkonsolidierte Böden resultieren die Parameter $\mathrm{c}^{\prime}=0 \mathrm{kPa}$ und $\varphi^{\prime}=\varphi_{\mathrm{c}}^{\prime}$, siehe Bild 3 (c) und (b).

In Bild 4 (b) wird die Festigkeitsumüllende der Barodesie für Ton [12] (Parameter lt. Tabelle 1) gezeigt. Die in Bild 3 qualitativ dargestellten Verhältnisse sind auch hier zu sehen. Die Linearisierung der Peakfestigkeiten gemäß Bild 4 (c) ergibt hier $\mathrm{q}_{0}=62 \mathrm{kPa}$, was für ein korrespondierendes Mohr-Coulomb Modell $c^{\prime}=29 \mathrm{kPa}$ bedeutet. Für den normalkonsolidierten Klei wird somit die 
Kohäsion von $15 \mathrm{kPa}$ nicht angesetzt. Dafür wird jedoch der Reibungswinkel von $\varphi^{\prime}=27^{\circ}$ auf $\varphi^{\prime}=30^{\circ}$ erhöht, um in einem gewünschten Spannungsbereich vergleichbare Festigkeiten zu erreichen, vgl. Bild 4 (a).

Für das Material werden in der EANG keine Laborversuche oder Quellen angegeben, weshalb die Parameter der Barodesie und der Hypoplastizität hier an simulierten Laborversuchen mit Hardening Soil kalibriert werden. Dazu werden mit Hardening Soil drainierte Triaxialversuche und ein isotroper Kompressionsversuch berechnet. Da bei Hardening Soil die Dichte nicht im Modell berücksichtigt wird, würde sich für eine andere Ausgangsdichte ein komplett unterschiedlicher Parametersatz ergeben, wohingegen die beiden anderen Modelle einen von der Dichte unhabhängien Parametersatz verwenden und die Dichteabhängigkeit über die Porenzahl gesteuert wird. Dies macht die Kalibrierung und die Vergleichbarkeit der Modelle schwierig, worauf nachfolgend eingegangen wird.

Die Modelle verwenden unterschiedliche Definitionen für die Überkonsolidierung (OCR), welche sich nicht allgemein ineinander umrechnen lassen. Für Hardening Soil ist die $\mathrm{OCR}$ mit $\mathrm{OCR}_{\sigma}=\frac{\sigma_{\mathrm{p}}^{\prime}}{\sigma_{\mathrm{zz}}^{\prime}}$ definiert [21]. Damit wird das Verhältnis der größten bisherigen effektiven Vertikalspannung $\sigma_{\mathrm{p}}^{\prime}$ zur aktuellen effektiven Vertikalpannung $\sigma_{\mathrm{zz}}^{\prime}$ verwendet. Bei den beiden anderen Modellen erhält man $\mathrm{OCR}_{\mathrm{p}}=\frac{\mathrm{p}_{\mathrm{e}}^{\prime}}{\mathrm{p}^{\prime}}$ in Bezug auf die isotrope Normalkonsolidierung (iso-NCL) als Verhältnis des equivalenten Drucks nach Hvorslev

$$
\mathrm{p}_{\mathrm{e}}^{\prime}=\exp \left(\frac{\mathrm{N}-\ln (1+\mathrm{e})}{\lambda^{*}}\right)
$$

zum aktuellen effektiven mittleren Druck $\mathrm{p}^{\prime}$, vgl. Bild 5. Im $\mathrm{K}_{0}$-Zustand liegt die dazugehörige oed-NCL (normal compression line für ödometrische Kompression) unterhalb der iso-NCL (normal compression line für isotrope Kompression) ([25], S.142). Somit ist hier für einen ödometrisch normalkonsolidierten Boden $\mathrm{OCR}_{\mathrm{p}}>1$.

Mit einer einheitlichen $O C R=O C R_{\sigma}=O_{C R}=1,7$ ergeben die Schubsteifigkeiten in den nachfolgend simulierten Versuchen eine gute Übereinstimmung. Nach den im vorherigen Abschnitt erläuterten Prinzipien der Critical States Soil Mechanics wird mit OCR $=1,7<2,0$ ein nur leicht überkonsolidiertes Materialverhalten abgebildet, womit die dort getroffenen Annahmen mit $c^{\prime}=0$ $\mathrm{kPa}$ und $\varphi^{\prime}=\varphi_{\mathrm{c}}^{\prime}=30^{\circ}$ gelten. Mit dieser OCR werden die nachfolgend erläuterten Versuche zur Kalibrierung der Materialmodelle durchgeführt. Die benötigte Ausgangsporenzahl lässt sich mit $\mathrm{p}_{\mathrm{e}}^{\prime}=\mathrm{OCR}_{\mathrm{p}} \cdot \mathrm{p}^{\prime}$ und Gleichung (1) zu e $=\exp \left(\mathrm{N}-\lambda^{*} \cdot \ln (\mathrm{OCR}\right.$. $\left.\left.\mathrm{p}^{\prime}\right)\right)-1$ ermitteln.

Bei dem isotropen Kompressionsversuch wird ausgehend von einer Spannung von $30 \mathrm{kPa}$ eine Belastung auf $300 \mathrm{kPa}$ und eine anschließende Entlastung auf $30 \mathrm{kPa}$ simuliert, siehe Bild 6. Diese Simulation wird zur Kalibrierung der Parameter $\lambda^{*}$ und $\kappa^{*}$ in der Barodesie und der Hypoplastizität genutzt. Da Hardening Soil keine Abhängigkeit von der Porenzahl beinhaltet kann der Parameter N der Barodesie und Hypoplastizität nicht an Simulationen kalibriert werden. Dieser Parameter kann hier jedoch zu 1 gesetzt werden, da die Porenzahl in den Simulationen aus der $\mathrm{OCR}_{\mathrm{p}}$ ermittelt wird.

Für die Hypoplastizität muss nun noch der Parameter $v$ an Triaxialversuchen bestimmt werden. Dazu werden drei Triaxialversuche mit einer Seitenspannung von $100 \mathrm{kPa}, 150 \mathrm{kPa}$ und $200 \mathrm{kPa}$ mit Hardening Soil simuliert, vgl. Bild 7. An den Simulationskurven ist zu erkennen, dass die maximale Vertikalspannung in allen drei Modellen gleich groß ist. Die volumetrischen Verzerrungen von Hypoplastizität und Barodesie sind ähnlich, allerdings fallen diese leicht geringer aus als bei Hardening Soil.

Zum abschließenden Vergleich wird mit den ermittelten Parametern auch noch ein Ödometerversuch simuliert, siehe Bild 8. Im Gegensatz zum isotropen Kompressionsversuch verhält sich Hardening Soil in dieser Simulation weicher als die beiden anderen Modelle.

Für Hardening Soil wird der in Tabelle 1 dargestellte Parametersatz verwendet, welcher bis auf $c^{\prime}$ und $\varphi^{\prime}$ der EANG entnommen ist. Die Materialparameter der Barordesie sowie der Hypoplastizität sind in Tabelle 2 gegeben. Zusätzlich wird eine Wichte $\gamma=15 \mathrm{kPa}$ und eine Sättigungswichte von $\gamma_{\mathrm{r}}=16 \mathrm{kPa}$ verwendet.

\subsection{Numerische Modellierung}

Für die Berechnungen wird das Finite Elemente-Programm PLAXIS [21] verwendet. Es wird ein ebener Verformungszustand 
angenommen. Das FE-Modell (Geometrie und Netz) wird von Beiblatt 1 der EANG [4] übernommen. Es hat eine Breite von 75 m, eine Tiefe von $30 \mathrm{~m}$ unter der Geländeoberkante, womit keine nennenswerten Spannungsänderungen bzw. Verformungen an den Rändern des Modells auftreten und die Empfehlungen der EANG [4] (Abschnitt 2.2) erfüllt werden. Das FE-Modell wird mit 1556 15-knotigen Dreieckselementen diskretisiert (Bild 9). Eine Netzverfeinerung auf die doppelte Anzahl von Elementen zeigt vernachlässigbare Unterschiede in den Berechnungsergebnissen. Die Spundwand wird durch Plattenelemente mit einer Dehnsteifigkeit von EA $=2,53 \cdot 10^{6} \mathrm{kN} / \mathrm{m}$, einer Biegesteifigkeit von EI $=3,02 \cdot 10^{4} \mathrm{kNm}^{2}$ und einer spezifischen Gewichtskraft von $\mathrm{w}=0,875 \mathrm{kN} / \mathrm{m}^{2}$ abgebildet. Die Steife weist eine Dehnsteifigkeit von $\mathrm{EA}=1,5 \cdot 10^{6} \mathrm{kN} / \mathrm{m}$ auf. Als Randbedingungen wird an den Seiten die Horizontalverschiebung und am Boden die Verschiebung in allen Raumrichtungen gesperrt.

Während des Aushubs wird der Boden unterhalb des Grundwassers als undrainiert, oberhalb als drainiert modelliert. Während des Aufbringens der Auflast im ersten Berechnungsschritt wird im gesamten Modell mit drainierten Bedingungen gerechnet. Das Wasser wird gemeinsam mit dem Boden ausgehoben. Der ursprüngliche Grundwasserspiegel bleibt dabei unverändert. Am Fuß der Spundwand stellt sich auf Grund der Gleichgewichtsbedingungen für jeden Aushubschritt ein gleicher Wasserdruck auf beiden Seiten ein. Zur Interaktion zwischen Boden und Strukturelementen wird in PLAXIS bei Verwendung eines UDSM eine ödometrische Interfacesteifigkeit benötigt. Diese lässt sich nach [21] anhand der ödometrischen Steifigkeit bei $\mathrm{p}_{\text {ref }}^{\prime}=100 \mathrm{kPa}$ ermitteln und ergibt für die Barodesie und die Hypoplastizität näherungsweise $\mathrm{E}_{\mathrm{oed}}^{100}=2000 \mathrm{kPa}$. Zur besseren Vergleichbarkeit wird für alle Materialmodelle der gleiche Ausgangsspannungszustand, ein Erdruhedruck mit $\mathrm{K}_{0}=1-\sin \varphi^{\prime}=1-\sin \varphi_{\mathrm{c}}^{\prime}$, angenommen.

\subsection{Berechnung der Verformungen}

Analog zu [4] wird die Berechnung in folgenden Schritten durchgeführt:

1. Ermittlung der Anfangsspannungen

2. Aufbringen der Verkehrslast

3. Aktivierung der Wand

4. Aushub 1 auf $-2,00 \mathrm{~m}$

5. Einbau der Steife auf $-1,50 \mathrm{~m}$

6. Aushub 2 auf $-4,00 \mathrm{~m}$

7. Aushub 3 auf $-6,00 \mathrm{~m}$

Der Einbau der Spundwand erfolgt „wished in place” und die Verformungen werden vor Beginn des Aushubs zurückgesetzt.

\subsection{Berechnung der Gesamtstandsicherheit}

In einem weiteren Schritt soll mittels Parameterreduktion die Gesamtstandsicherheit des Baugrubenverbaus ermittelt werden. Die Standsicherheitsberechnung mit dem Hardening Soil Modell ist bereits standardmäßig in PLAXIS implementiert. Hier wird eine $\varphi^{\prime}-$ $\mathrm{c}^{\prime}$-Reduktion bis zum Eintreten des Versagenszustands durchgeführt. Die erreichte Sicherheit $\eta$ ergibt $\operatorname{sich} \operatorname{dann} \operatorname{zu} \eta=\frac{\tan \varphi^{\prime}}{\tan \varphi_{\text {red }}^{\prime}}=$ $\frac{\mathrm{c}^{\prime}}{\mathrm{c}_{\text {red }}^{\prime}}$

Ein analoges Verfahren zur Parameterreduktion mit der Barodesie wird in [19] vorgestellt. Um eine starke Überkonsolidierung des Bodens zu berücksichtigen, wird dort zusätzlich zum kritischen Reibungswinkel $\varphi_{c}^{\prime}$ der Parameter N reduziert. Da es sich in diesem Fall um einen nur leicht überkonsolidierten Boden handelt, wird hier bei der Barodesie nur der kritische Reibungswinkel mit $\tan \varphi_{\mathrm{c}, \mathrm{red}}^{\prime}=\frac{\tan \varphi_{\mathrm{c}}^{\prime}}{\eta}$ reduziert, bis sich ein Versagenszustand einstellt. Bei beiden Modellen wird der Reibungswinkel auf die gleiche Weise reduziert, weshalb auch die Sicherheitsdefinitionen vergleichbar sind.

Für die Hypoplastizität ist aktuell noch kein Verfahren zur Reduktion der Materialparameter verfügbar und somit wird die Standsicherheitsberechnung nur für die Barodesie und Hardening Soil durchgeführt. Eine Reduktion der Materialparameter der 
Strukturelemente wird hier nicht berücksichtigt, da nur die Unterschiede der verschiedenen Materialmodelle für den Boden betrachtet werden.

\subsection{Berechnungsergebnisse}

\subsubsection{Aushub der Baugrube}

Die durch den Aushub auftretenden Verformungen sind in Bild 10 bis 12 für die verschiedenen Materialmodelle dargestellt. Bei allen Modellen zeigt sich eine in unmittelbarer Nähe zur Spundwand ausbildende Setzungsmulde (Bild 10). Mit Hardening Soil fällt diese Mulde deutlich flacher aus als mit den anderen Modellen.

Neben Setzungen an der Geländeoberfläche treten durch die Entlastung des Bodens Hebungen an der Baugrubensohle auf (Bild 11). Diese liegen für alle Modelle in einer ähnlichen Größenordnung. Hierbei fallen mit der Barodesie die prognostizierten Hebungen am geringsten aus.

Mit Hardening Soil und der Barodesie wird eine nahezu gleich große Horizontalverformung der Spundwand ermittelt. Mit der Hypoplastizität fällt diese etwas größer aus (Bild 12). Beim auftretenden Biegemoment zeigt sich ein ähnliches Bild.

Ein weiterer wichtiger Punkt für die Bemessung einer Spundwand ist die maximal zu erwartende Normalkraft in der Steife. Hier treten bei der Barodesie mit $143 \mathrm{kN}$ und der Hypoplastizität mit $140 \mathrm{kN}$ sehr ähnliche Normalkräfte auf. Bei Hardening Soil fällt diese hier mit $120 \mathrm{kN}$ etwas geringer aus.

\subsubsection{Gesamtstandsicherheit}

Die Ergebnisse der Parameterreduktion zeigen deutliche Unterschiede in der ermittelten Sicherheit. Mit Hardening Soil tritt Versagen nach einer Reduktion der Materialparameter bis $\eta=1,48$ auf. Mit der Barodesie ist nur eine Reduktion bis zu $\eta=1,28$ möglich, bis sich ein Versagenszustand einstellt. Wie bereits erwähnt ist für die Hypoplastizität derzeit noch kein Verfahren zur Reduktion der Materialparameter verfügbar.

Zur Visualisierung der Versagenszustands sind in Bild 13 die deviatorischen Verzerrungen dargestellt. Die Größe dieser Verzerrungen ist hier nach Eintreten des Versagens ohne Bedeutung. Es ist jedoch zu erkennen, dass es hierbei zu einer Konzentration der Scherverformungen kommt.

\section{$4 \quad$ Diskussion der Ergebnisse}

\subsection{Verformungsprognosen}

Ein Vergleich der Berechnungsergebnisse zeigt, dass sich hier bei einem ähnlichen Verhalten der mit den verschiedenen Materialmodellen simulierten Laborversuche, ebenso in der FE-Berechnung ähnliche Verformungen und daraus resultierende Schnittgrößen in den Stützkonstruktionen ergeben. Bei den Beispielen aus der EANG [4] an der gleichen Geometrie wird aufgezeigt, dass sich bei der Verwendung einfacherer Materialmodelle, wie linear elastische Modelle mit Mohr Coulomb, auch qualitativ sehr große Unterschiede in den Verformungen auftreten können. Es ist somit erforderlich, insbesondere bei undrainierten Berechnungen in effektiven Spannungen, auf höherwertige Materialmodelle zurückzugreifen. In diesem Fall sind zur Verformungsberechnung alle drei Modelle gleichermaßen geeignet.

Andere Untersuchungen, beispielsweise von Herle und Nitzsche [26] für Böschungen oder von Schranz [27], zeigen jedoch, dass in anderen Fällen selbst bei ähnlichen Ergebnissen von simulierten Laborversuchen deutliche Unterschiede in den Verformungen bei den FE-Berechnungen auftreten können. Es kann hierfür also keine generell gültige Antwort gegeben werden und das Verhalten der Materialmodelle muss in jedem Fall einzeln untersucht werden.

\subsection{Standsicherheitsberechnung}


Überraschend sind hier nun jedoch die Ergebnisse der Parameterreduktion, da diese einen deutlichen Unterschied in den prognostizieren Standsicherheiten des Baugrubenverbaus aufweisen. Hierfür heißt es in der EANG, dass die ermittelte Sicherheit nur wenig von der Wahl des Materialmodells abhängt und dass der Spannungspfad hierbei nur eine untergeordnete Rolle hat [4]. Es ist an dieser Stelle jedoch anzumerken, dass in der EANG nur Modelle mit einer Mohr-Coulomb Grenzbedingung verglichen werden. Um den Einfluss des Materialmodells genauer zu betrachen, wird in einem Punkt, welcher auf der sich ausbildenden Scherfuge liegt (Bild 13) die Entwicklung des Spannungspfades betrachtet. Für weitere Punkte auf der Scherfuge, sowohl auf der aktiven als auch auf der passiven Seite, zeigen sich qualitativ gleiche Unterschiede im Verhalten der Modelle.

In Bild 16 werden die Spannungspfade mit den Deviatorspannungen $\mathrm{q}=\sqrt{2 / 3}\|\mathbf{s}\|$ über den mittleren effektiven Druck $\mathrm{p}^{\prime}=$ $\left(\sigma_{1}^{\prime}+\sigma_{2}^{\prime}+\sigma_{3}^{\prime}\right) / 3$ aufgetragen. Mit dem Tensor der Deviatorspannungen $\mathbf{s}=\boldsymbol{\sigma}^{\prime}-\mathbf{1} \cdot \mathrm{p}^{\prime}$ und der Norm $\|\mathbf{s}\|=\sqrt{\operatorname{tr}\left(\mathbf{s}^{\mathrm{T}} \cdot \mathbf{s}\right)}$, wobei mit $\operatorname{tr}(\mathbf{A})=\mathrm{A}_{11}+\mathrm{A}_{22}+\mathrm{A}_{33}$ die Spur eines Tensors bezeichnet wird.

Alle drei Modelle starten im gleichen $\mathrm{K}_{0}$-Zustand. Das Aufbringen der Auflast bei drainierten Verhältnissen führt zu einem Anstieg sowohl des mittleren Drucks als auch der Deviatorspannungen. Der Aushub erfolgt undrainiert und bis zum Einbau der Steife nach dem ersten Aushubschritt kommt es zu einem Abfallen der Deviatorspannungen, anschließend zu einem Anstieg. Während bei der Barodesie und der Hypoplastizität der mittlere Druck durch den Aushub abfällt, bleibt dieser bei Hardening Soil näherungsweise konstant. Der Spannungspfad der Berechnung mit der Barodesie weist zum Ende des Aushubs ein etwas höheres Verhältnis q/p' auf als in einer Berechnung mit Hardening Soil, was auf eine etwas geringere Sicherheit hin deutet, jedoch noch nicht die großen Unterschiede in der ermittelten Standsicherheit erklären kann. Da bei einem ebenen Verzerrungszustand in einem $\mathrm{p}^{\prime}$-q-Diagramm nicht alle Informationen der Spannungspfade dargestellt werden können, müssen diese im Detail in der Deviatorebene betrachtet werden.

\subsection{Betrachtung der Deviatorebene}

Die Unterschiede in den Spannungspfaden lassen sich anschaulich in der normierten Deviatorebene aufzeigen. Dabei wird die aufgetragene Hauptspannung durch den mittleren Druck p' dividiert, womit der gesamte Spannungspfad in eine deviatorische Ebene projeziert wird.

Während Hardening Soil das Versagenskriterium nach Mohr-Coulomb zugrunde liegt, wird mit der Hypoplastizität sowie der Barodesie die Grenzbedingung nach Matsuoka-Nakai abgebildet (Bild 15). Diese Grenzbedinungen stimmen für axialsymmetrische Zustände überein. Mit Matsuoka-Nakai sind außerhalb der axialsymmetrischen Zustände jedoch höhere Deviatorspannungen als bei Mohr-Coulomb möglich

Zur Beschreibung der Lage des Spannungspfades in der Deviatorebene wird der Lode Winkel $\theta$ mit

$$
\cos (3 \theta)=\frac{\operatorname{det}(\mathbf{s})}{2}\left(\frac{6}{\|\mathbf{s}\|^{2}}\right)^{3 / 2}
$$

verwendet, welcher hier für axialsymetrische Kompression $\sigma_{1}^{\prime}>\sigma_{2}^{\prime}=\sigma_{3}^{\prime}$ bei $\theta=60^{\circ}$ und für axialsymetrische Extension $\sigma_{3}^{\prime}<$ $\sigma_{1}^{\prime}=\sigma_{2}^{\prime}$ bei $\theta=0^{\circ}$ liegt (Bild 15). Es hat sich gezeigt, dass sich bei Problemen mit ebenem Verzerrungszustand Grenzspannungen ergeben, welche in der Deviatorebene in einen Bereich zwischen $\theta=30^{\circ}$ und $\theta=45^{\circ}$ liegen [28]. Auf Grund der Mohr-Coulomb Grenzbedingung sollten für Hardening Soil in diesem Bereich die aufnehmbaren Deviatorspannungen im Verhältnis zum mittleren Druck geringer ausfallen als mit der Barodesie.

In Bild 16 werden die normierten Spannungspfade dargestellt. Es ist zu erkennen, dass die Spannungspfade der Barodesie und der Hypoplastizität im axialsymmetrsichen Zustand starten und während des Aushubs in den Bereich zwischen $\theta=30^{\circ}$ und $\theta=45^{\circ}$ streben, wohingegen Hardening Soil gegen Ende des Aushubs auf der $\sigma_{1}^{\prime} / \mathrm{p}^{\prime}$-Achse landet. Dort sind die maximal mobilisierbaren Deviatorspannungen am größten und somit ist der Abstand des Spannungszustands zur Grenzbedingung bei Hardening Soil deutlich größer als bei der Barodesie.

Der mittels Hypolastizität ermittelte Spannungspfad liegt zum Ende des Aushubs außerhalb der Grenzbedingung für den kritischen 
Zustand. Auf Grund der Änderung des mittleren Drucks bei undrainierten Verhältnissen im Bereich unterhalb der Baugrubensohle stellt sich dort eine OCR zwischen 2,4 und 2,9 ein. Damit handelt es sich hierbei nun um einen stark überkonsolidierten Boden, bei welchem der mobilisierbare Reibungswinkel höher ist als der kritsiche Reibungswinkel $\varphi_{c}^{\prime}$.

Zur genaueren Betrachtung werden mit den Modellen normalkonsolidierte Biaxialversuche ausgehend vom $\mathrm{K}_{0}$-Zustand simuliert (Bild 17). Wie sich zeigt, streben die mit der Barodesie und der Hypoplastizität erhaltenen Spannungspfade in den Bereich für ebene Verzerrungszustände, wohingegen die Pfade des drainierten und undrainierten Biaxialversuchs mit Hardening Soil annähernd auf der $\sigma_{1}^{\prime} / \mathrm{p}^{\prime}$-Achse bleiben.

Ein Vergleich mit Biaxialversuchen mit normalkonsolidiertem Ton nach [29] (Bild 17) zeigt, dass mit der Barodesie sowie der Hypoplastizität hier ein qualitativ richtiges Verhalten abgebildet wird. Die Abbildbarkeit dieses Verhaltens mit der Barodesie lässt sich durch die dort implementierte Spannungs-Dilatanz Beziehung erklären, vgl. [30].

Eine Betrachtung der Spannungspfade zeigt, dass eine Trennung von Verformungsberechnung und Standsicherheitsberechnung zumindest im Allgemeinen nicht möglich ist. Die ermittelte Standsicherheit hängt in diesem Fall maßgeblich von dem zu Beginn der Standsicherheitsberechnung, d.h. vor der rechnerischen Reduktion der Scherfestigkeit, vorherrschenden Spannungszustand ab, welcher wiederum ein Ergebnis der Verformungsberechnung ist.

\section{$5 \quad$ Zusammenfassung}

Es wurden Simulationen eines Baugrubenverbaus in weichem Boden mit unterschiedlichen, höherwertigen Materialmodellen durchgeführt. Verwendet wurden neben der Barodesie ein elstoplastisches Materialmodell, Hardening Soil und das hypoplastische Materialmodell für Ton von Mašín.

Dabei wurde festgestellt, dass bei dieser Problemstellung, bei sorgfältiger Kalibrierung der Materialparameter und konsistent er Wahl der Anfangszustände (Spannung, Porenzahl und OCR), alle Modelle ähnliche Verformungen prognostizieren. Große Unterschiede zeigen sich jedoch in den ermittelten Standsicherheiten mittels Reduktion der Scherfestigkeit. Dabei zeigt sich, dass die mit Hardening Soil ermittelte Sicherheit deutlich höher ausfällt als mit der Barodesie. Diese Unterschiede können zum Teil auf die Spannungspfade zurückgeführt werden, welche sich aufgrund des ebenen Verformungszustandes einstellen. Hier ist also die Trennung von Verformungs- und Standsicherheitsberechnungen nicht möglich. Die Barodesie ist, wie auch die Hypoplastizität, in der Lage das Verhalten des Bodens im ebenen Verzerrungszustand realistisch abzubilden und ist somit für ebensolche Randbedingungen bei Finite Elemente Berechnungen gut geeignet. Anhand dieser Untersuchungen kann nicht gesagt werden, welches Materialmodell das Bodenverhalten im Gesamten besser abbildet. Generell ist es sehr wichtig, sich mit dem für die Berechnung verwendeten Materialmodell auseinanderzusetzen, da nur so eine korrekte Interpretation der Ergebnisse möglich ist.

\section{Danksagung}

Die Arbeit von M. Bode und G. Medicus wurde durch den Fonds zur Förderung der wissenschaftlichen Forschung unterstüzt (Austrian Science Fund (FWF): P 28934-N32). Wir danken den beiden anoynmen Gutachtern für die sorgfältige Durchsicht der Einreichung und der für die Klarheit und Schlüssigkeit der Darstellung wertvollen Kommentare.

\section{Danksagung}

Das AutorInnenteam erstellte das Konzept der Arbeit. M.B. führte die Finite Elemente Berechnungen durch und wertete diese aus. G.M. und W.F. lieferten die Grundlagen zur Ermittlung der Materialparameter. F.S. kalibrierte die verwendeten Materialmodelle und führte die Berechnungen der Elementversuche durch. Alle Autoren und die Autorin diskutierten die Ergebnisse und trugen zu dem entsprechenden Abschnitt bei. Der Text wurde federführend von M.B. erstellt, mit einzelnen Text- und Bildbeiträgen (jeweils zu den zuvor erwähnten Themen) der anderen Autoren und der Autorin.

\section{References}


[1] Benz, T., Bergholz, K.: Geotechnische Materialmodelle im Spannungsfeld zwischen Forschung und Praxis. BAW Mitteilungen Karlsruhe: Bundesanstalt für Wasserbau (BAW), (101):75-87, 2017.

[2] Schweiger, H., Vermeer, P.A., Markus W.: On the design of deep excavations based on finite element analysis. Zur Bemessung tiefer Baugruben mit der Finite-Elemente-Methode. Geomechanics and Tunnelling, 2(4):333-344, 2009.

[3] Lim, A., Ou, C., Hsieh, P.: Evaluation of clay constitutive models for analysis of deep excavation under undrained conditions. Journal of GeoEngineering, 5(1):9-20, 2010.

[4] Deutsche Gesellschaft für Geotechnik e.V.: Empfehlungen des Arbeitskreises Numerik in der Geotechnik - EANG. Ernst W. + Sohn Verlag, 2014.

[5] Mitew-Czajewska, M: Evaluation of hypoplastic clay model for deep excavation modelling. Archives of Civil Engineering, 62(4):73-86, 2016.

[6] Mašín, D.: A hypoplastic constitutive model for clays. International Journal for Numerical and Analytical Methods in Geomechanics, 29(4):311-336, 2005.

[7]Tschuchnigg, F., Schweiger, H., Sloan, S., Lyamin, A., Raissakis, I.: Comparison of finite-element limit analysis and strength reduction techniques. Géotechnique, 65(4):249-257, 2015.

[8] Kolymbas, D., Fellin, W., Schneider-Muntau, B., Medicus, G. Schranz, F.: Zur Rolle der Materialmodelle beim Standsicherheitsnachweis. geotechnik, 39(2):89-97, 2016.

[9] $\mathrm{Lim}, \mathrm{A}$., $\mathrm{Ou}, \mathrm{C}$.: Stress paths in deep excavations under undrained conditions and its influence on deformation analysis. Tunnelling and Underground Space Technology, 63:118-132, 2017.

[10] Schanz, T., Vermeer, P.A., Bonnier, P.G.: The hardening soil model: formulation and verification. Beyond 2000 in computational geotechnics, :281-296, 1999.

[11] Mašin, D.: Clay hypoplasticity with explicitly defined asymptotic states. Acta Geotechnica, 8(5):481-496, 2013.

[12] Medicus, G., Fellin, W.: An improved version of barodesy for clay. Acta Geotechnica, 12(2):365-376, 2017.

[13] Kolymbas, D.: Barodesy as a novel hypoplastic constitutive theory based on the asymptotic behaviour of sand. geotechnik, 35(3):187-197, 2012.

[14] Kolymbas, D.: Introduction to barodesy. Géotechnique, 65(1):52-65, 2015.

[15] Gudehus, G., Amorosi, A., Gens, A., Herle, I., Kolymbas, D., Mašín, D., Muir Wood, D., Niemunis, A., Nova, R., Pastor, M., u.a.: The soilmodels. info project. International Journal for Numerical and Analytical Methods in Geomechanics, 32(12):1571-1572, 2008.

[16] Medicus, G., Fellin, W., Schranz, F.: Konzepte der Barodesie. Bautechnik, 95(9):620-638, 2018.

[17] Fellin, W.,Ostermann, A.: Consistent tangent operators for constitutive rate equations. International Journal for Numerical and Analytical Methods in Geomechanics, 26(12):1213-1233, 2002.

[18] Schneider-Muntau, B., Chen, C., Bathaeian, I.: Simulation of shear bands with Soft PARticle Code (SPARC) and FE. GEM International Journal on Geomathematics, 8(1):135-151, 2017.

[19] Schneider-Muntau, B., Medicus, G., Fellin, W.: Strength reduction method in Barodesy. Computers and Geotechnics, 95:57 - 67, 2018.

[20] Bode, M.: Anwendung der Barodesie für Ton bei Finite Elemente Berechnungen. Masterarbeit, Universität Innsbruck, 2017.

[21] Binkgreve, R.B.J., Kumarswamy, S., Swolfs W.M.: Plaxis 2D 2016 Manual. PLAXIS BV, Delft, 2017.

[22] Krey, H.: Rutschgefährliche und fließende Bodenarten. Bautechnik, 5(1927):485-489, 1927. 
[23] Tiedemann, B.: Über die Schubfestigkeit bindiger Böden. Bautechnik, 15:400-403, 433-435, 1937.

[24] Bergholz, K., Herle, I.: Experimentelle Bestimmung der Nichtlinearität von Spannungsgrenzbedingungen im Bereich geringer Spannungen. geotechnik, 40(2):119-125, 2017.

[25] Wood, D.: Soil Behaviour and Critical State Soil Mechanics. Cambridge University Press, 1991.

[26] Herle, I., Nitzsche, K.: Prognose von aushubbedingten Baugrundverformungen. 16. Dresdner Baustatik-Seminar, 2012.

[27] Schranz, F.: Applications and developments of Barodesy. Dissertation, Universität Innsbruck, 2018.

[28] Nakai, T.: Modeling of soil behavior based on tij concept. Proc. of 13th Asian Regional Conf. on Soil Mech. and Geotechnical Eng., Keynote Paper, Kolkata, pages 69-89, 2007.

[29] Matsuoka, H., Sun, D.: The SMP concept-based 3D constitutive models for geomaterials. CRC Press, 2014.

[30] Medicus, G., Kolymbas, D., Fellin, W.: Proportional stress and strain paths in barodesy. International Journal for Numerical and Analytical Methods in Geomechanics, 40(4):509-522, 2016.

Autoren

Manuel Bode

AB Geotechnik und Tunnelbau

Universität Innsbruck

Technikerstraße 13

6020 Innsbruck

Manuel.Bode@uibk.ac.at

Fabian Schranz

Department of Engineering Science

University of Oxford,

Parks Road,

Oxford OX1 3PJ

Fabian.Schranz@eng.ox.ac.uk

Getraud Medicus

AB Geotechnik und Tunnelbau

Universität Innsbruck

Technikerstraße 13

6020 Innsbruck

Getraud.Medicus@uibk.ac.at

Wolfgang Fellin

AB Geotechnik und Tunnelbau

Universität Innsbruck

Technikerstraße 13

6020 Innsbruck

Wolfgang.Fellin@uibk.ac.at 

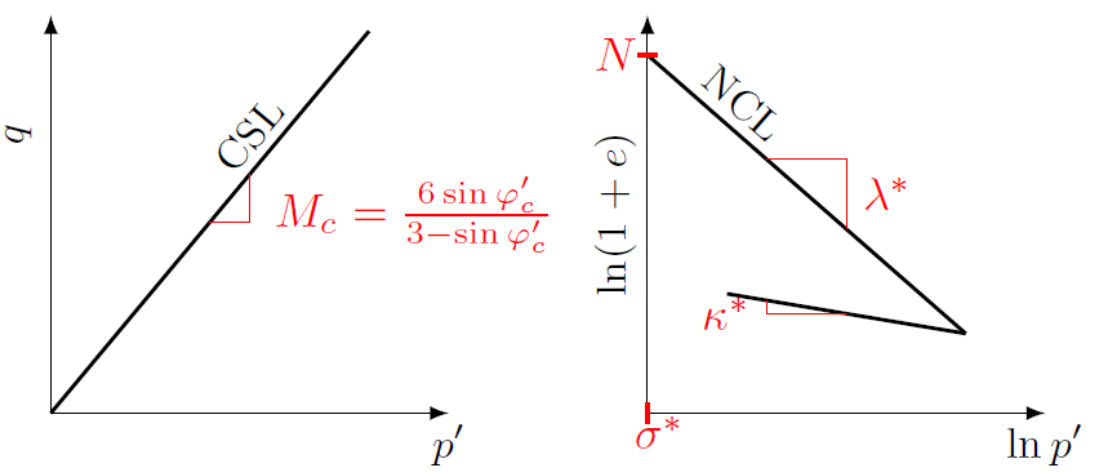

Bild 1 Materialparameter der Barodesie für Ton nach [12]. Für axialsymmetrische Zustände gilt $p^{\prime}=\frac{1}{3}\left(\sigma_{1}^{\prime}+2 \sigma_{2}^{\prime}\right)$ und $q=\sigma_{1}^{\prime}-\sigma_{2}^{\prime}$.

Fig. 1 Material parameters of barodesy for clay according to [12]. For axial symmetric states $p^{\prime}=\frac{1}{3}\left(\sigma_{1}^{\prime}+2 \sigma_{2}^{\prime}\right)$ and $q=\sigma_{1}^{\prime}-\sigma_{2}^{\prime}$.

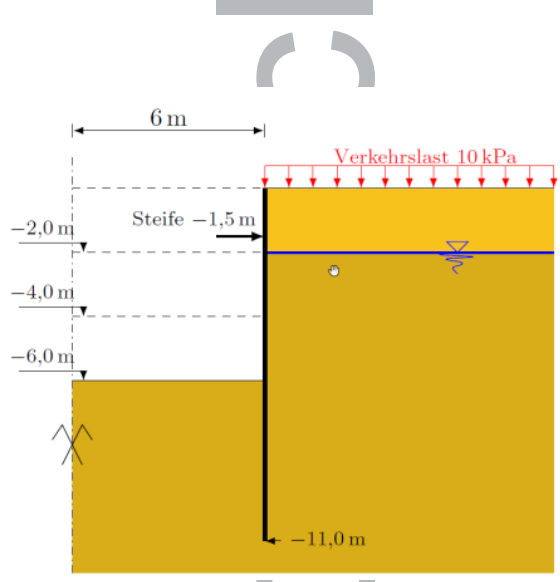

Bild 2 Systemskizze der berechneten Baugrube. Geometrie nach [4]

Fig. 2 Schematic sketch of the calculated excavation pit. Geometry according to [4]

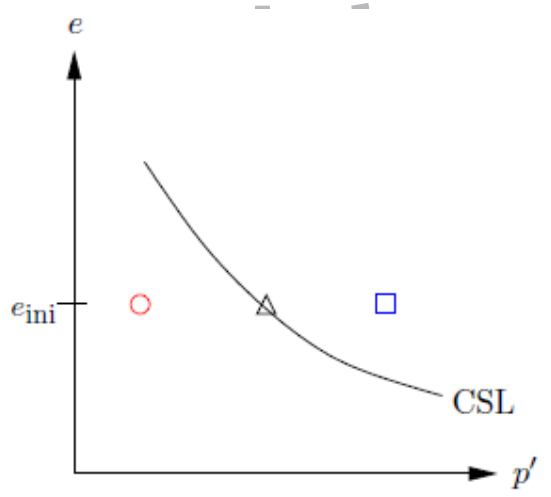

(a)

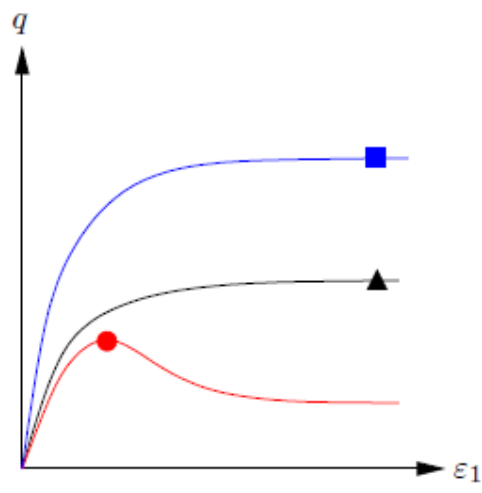

(b)

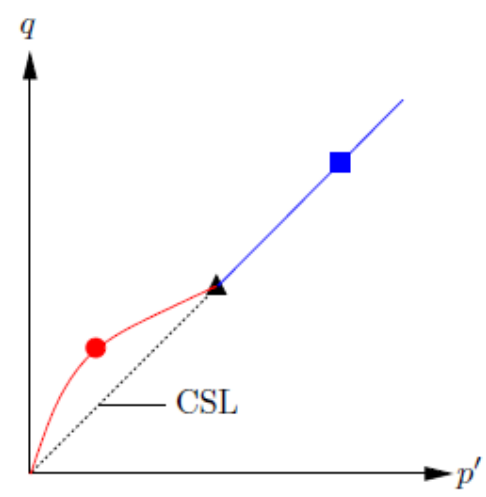

(c)

Bild 3 Scherfestigkeit in Triaxialversuchen: Die Anfangszustände sind mit offenen Symbolen, Peakzustände dränierter Triaxialversuche sind mit gefülten Symbolen gekennzeichnet. (a) Die CSL trennt dichten (०) von lockerem (匹) Boden im Anfangszustand. (b) Dichter Boden hat im Spannungs-Stauchungs Digramm einen Peak (•) mit anschließender Entfestigung. Bei lockerem Boden nimmt die Steifigkeit bis zum Erreichen des Peakzustandes kontinuierlich ab (匹). (c) Peakzustände (überhöht dargestellt) von dichtem Boden (•) liegen über der sog. Critical State Line (CSL), die für lockere Böden ( $\mathbf{\square}$ ) maßgebend ist.

Fig. 3 Shear strength in triaxial tests: The initial states are marked with open symbols, peak states of drained triaxial tests are marked with filled symbols. (a) The CSL separates dense (o) from loose ( $\mathbf{m})$ soil in the initial state. (b) Dense soil has a peak $(\cdot)$ in the stress compression digram with subsequent softening. For loose soil, the stiffness decreases continuously until the peak condition is reached $(\mathbf{-})$. (c) Peak conditions (exaggerated) of dense soil (•) lie above the Critical State Line (CSL), which is decisive for loose soil $(\mathbf{\square})$. 


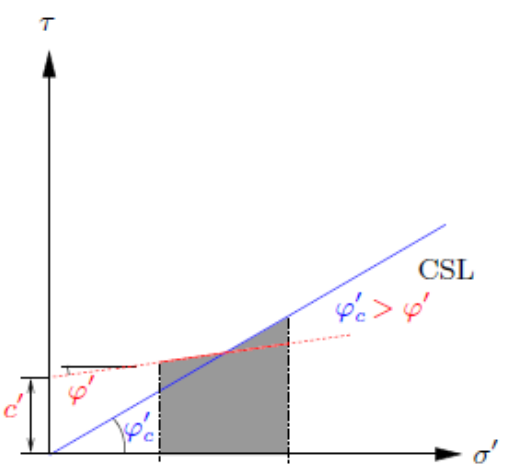

(a)

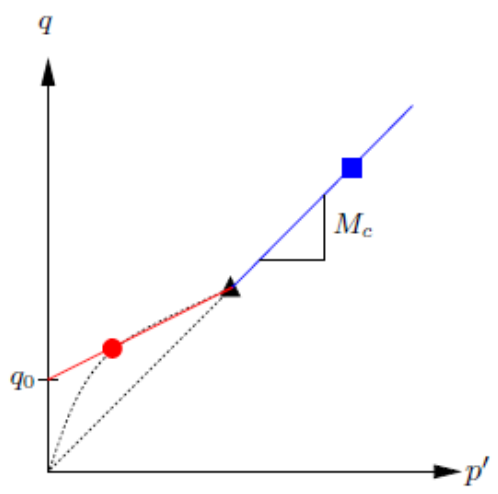

(b)

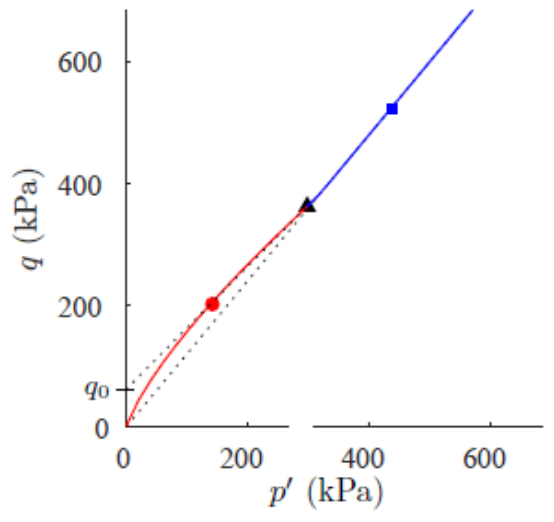

(c)

Bild 4 Peakzustände: a) Vergleichbare Festigkeiten zufolge $c^{\prime}$ und $\varphi^{\prime}$ bzw. $\varphi_{c}^{\prime}$; (b) Konzept nach Krey-Tiedemann [22], [23]; (c) Festigkeitsumhüllende der Barodesie $\mathrm{e}_{\mathrm{ini}}=0,95$

Fig. 4 Peak states: a) Comparable strengths based on $c^{\prime}$ and $\varphi^{\prime}$ and $\varphi_{c}^{\prime}$ respectively; (b) Concept after Krey-Tiedemann [22], [23]; (c) Strength envelope of Barodesy $\mathrm{e}_{\mathrm{ini}}=0,95$

口

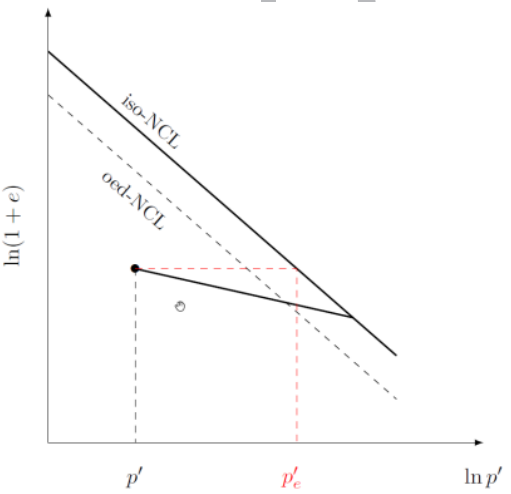

Bild 5 Schematische Darstellung der Definition der $\mathrm{OCR}_{\mathrm{p}}$ mit dem equivalenten Druck nach Hvorslev (1)

Fig .5 Schematic illustration of the definition of $\mathrm{OCR}_{\mathrm{p}}$ using the equivalent pressure of Hvorslev (1)

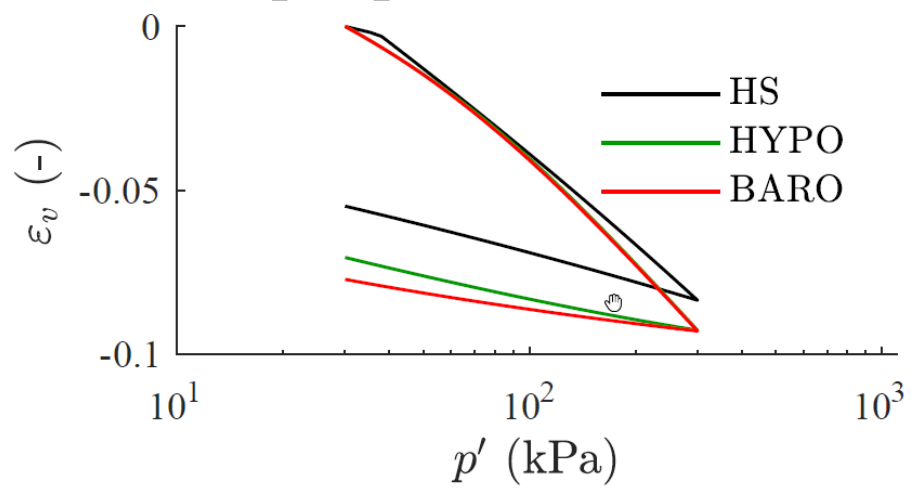

Bild 6 Isotroper Kompressionsversuch zur Kalibrierung der Materialmodelle mit $\mathrm{e}_{\mathrm{ini}}=1,216$ bzw. OCR $=1,7$

Fig. 6. Isotropic compression test used for calibration oft he models with $\mathrm{e}_{\mathrm{ini}}=1,216$ or $\mathrm{OCR}=1,7$ 

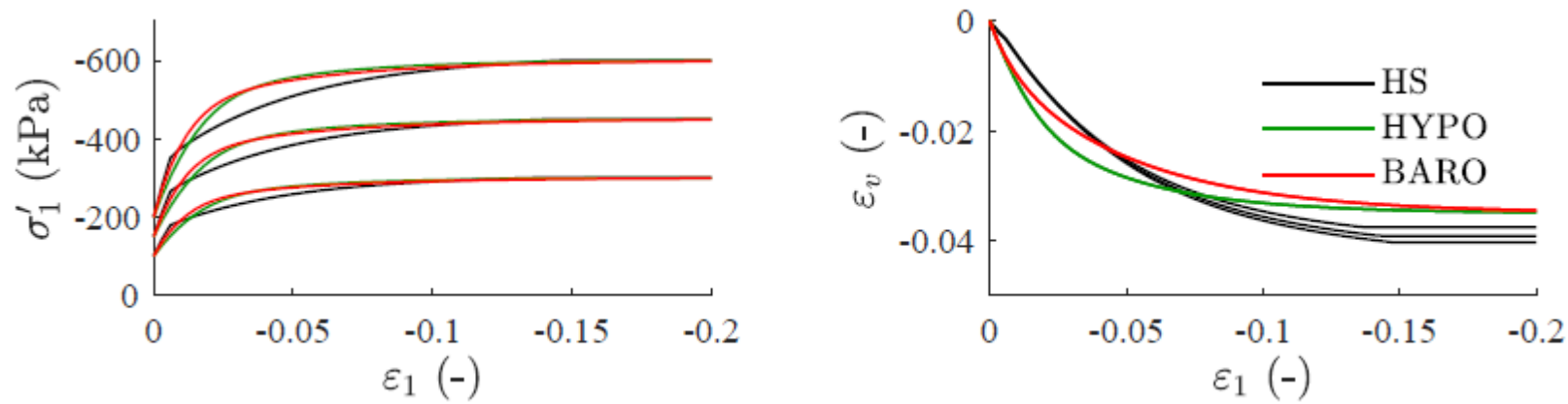

Bild 7 Simulierte drainierte Triaxialversuche zur Kalibrierung der Barodesie und der Hypoplastizität mit $O C R=1,7$

Fig. 7 Simulated drained triaxial tests used for calibration of Barodesy and Hypoplasticity with $O C R=1,7$

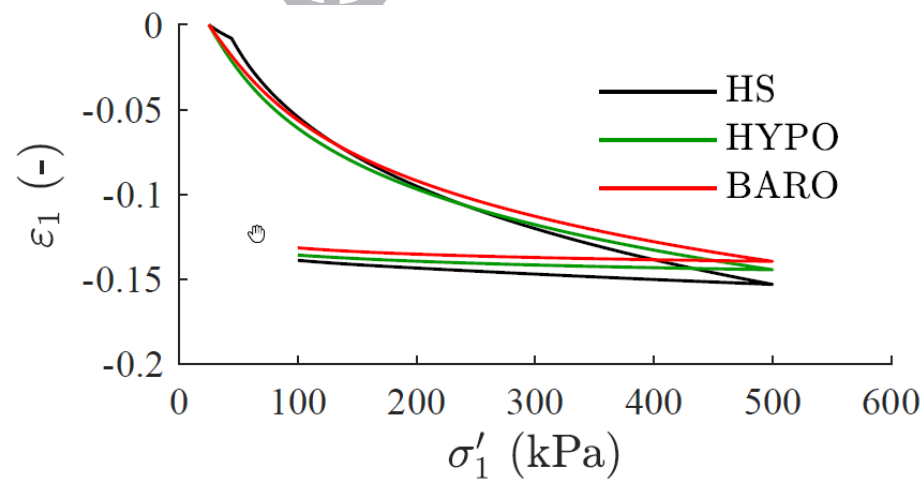

Bild 8 Ödometerversuch zur Kontrolle der Kalibrierung mit $\mathrm{e}_{\mathrm{ini}}=1,285$ bzw. OCR $=1,7$

Fig. 8 Oedometer test to verify the calibration with $\mathrm{e}_{\text {ini }}=1,285$ or. $\mathrm{OCR}=1,7$

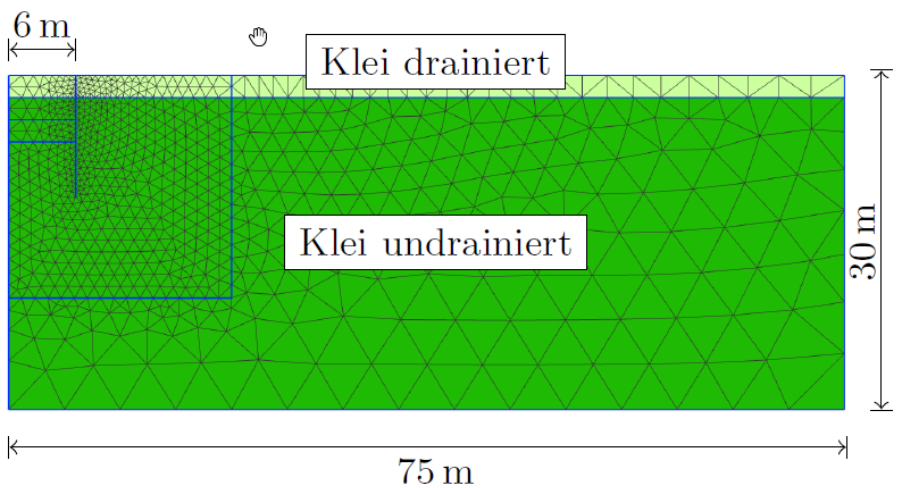

Bild 9 Abmessung des verwendeten FE-Modells mit 1556 15-knotigen Elementen nach [4]

Fig. 9 Dimension of the used FE-model with 1556 15-node elements according to [4] 


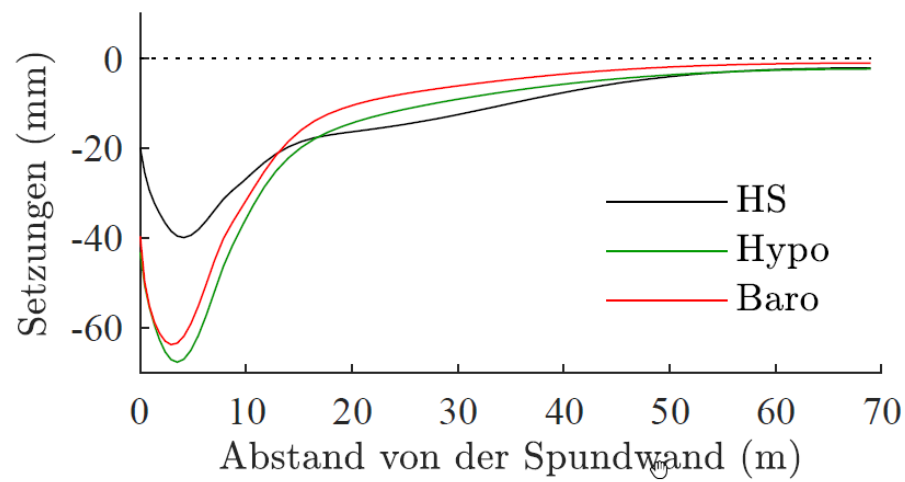

Bild 10 Neben der Baugrube auftretende Oberflächensetzungen zum Ende des Aushubs

Fig. 10 Surface settlement occurring next to the excavation pit at the end of excavation

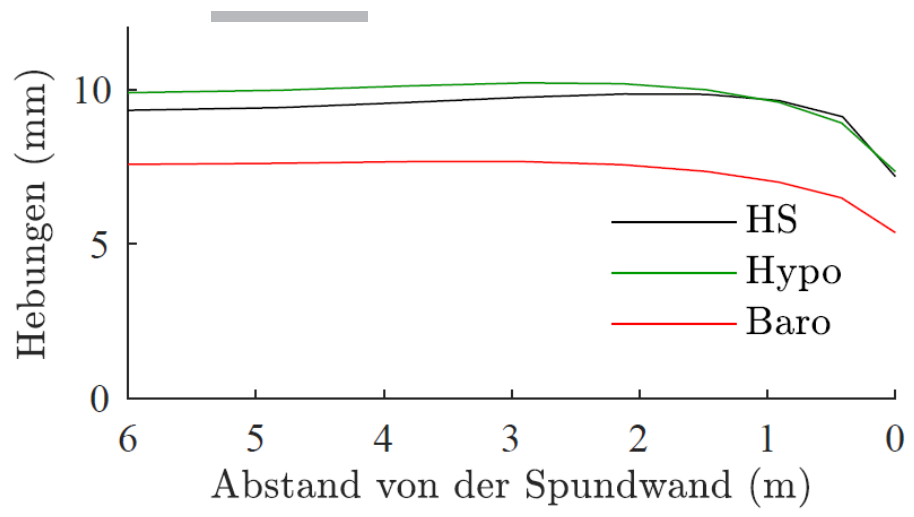

Bild 11 Hebungen der Baugrubensohle zum Ende des Aushubs

Fig. 11 Lifting of the bottom of the excavation pit at the end of excavation
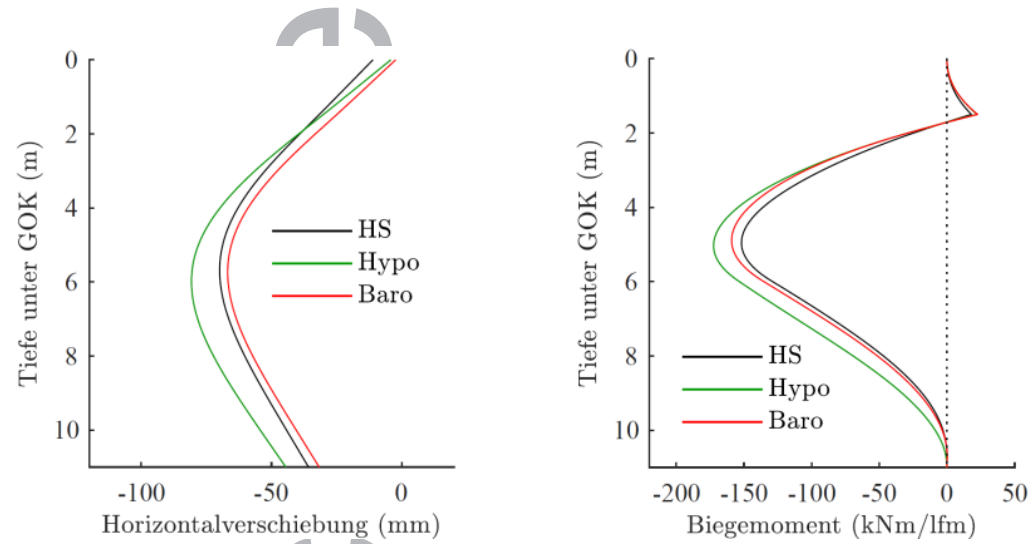

Bild 12 Horizontalverschiebung (links) und Biegemoment (rechts) der Spundwand zum Ende des Aushubs

Fig. 12 Horizontal displacement (left) and bending moment on the sheet pile wall (right) at the end of excavation 


\section{geotechnik Fachaufsatz/Bericht}

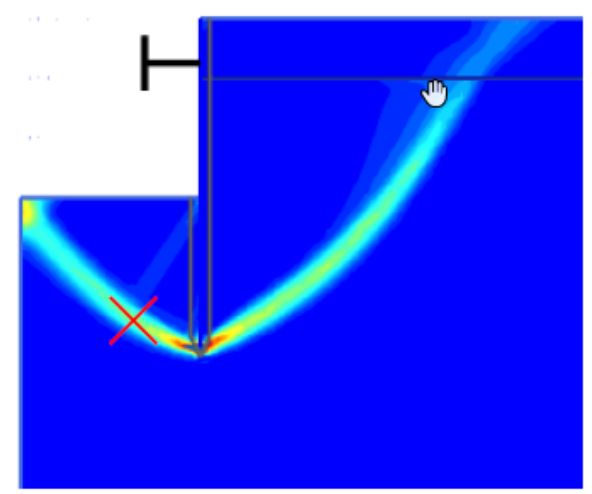

Bild 13 Beim Versagen auftretende Scherfuge, dargestellt über die deviatorischen Verzerrungen. Links: Barodesie; rechts: Hardening Soil. Mit $\times$ ist der Punkt zur Auswertung der Spannungspfade gekennzeichnet.

Fig. 13 Shear plane occurring during failure, represented by the deviatoric strains. Left: Barodesy; right: Hardening Soil. The point for the evaluation of the stress paths is marked with $\times$.

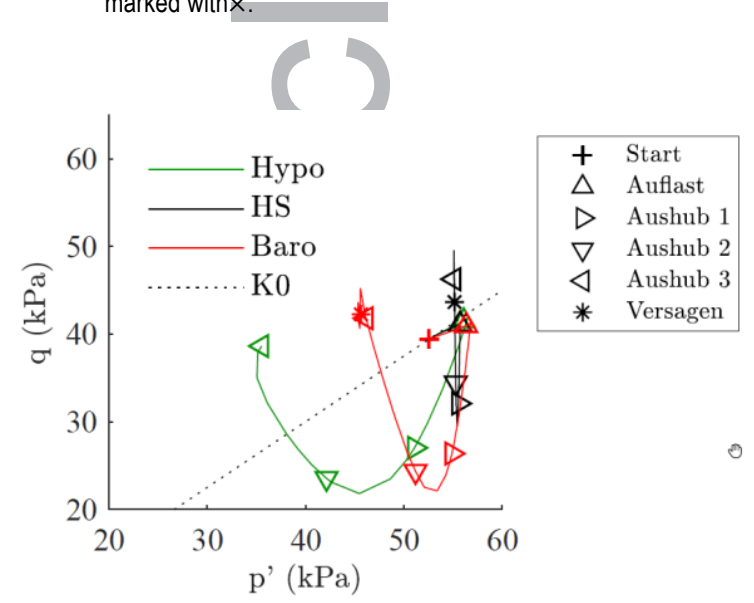

Bild 14 Darstellung der Spannungspfade im $\mathrm{p}^{\prime}$-q-Diagramm für den in Bild 13 markierten Punkt

Fig. 14 Representation of the stress paths in the $p^{\prime}-q$ plot for the point marked in Figure 13

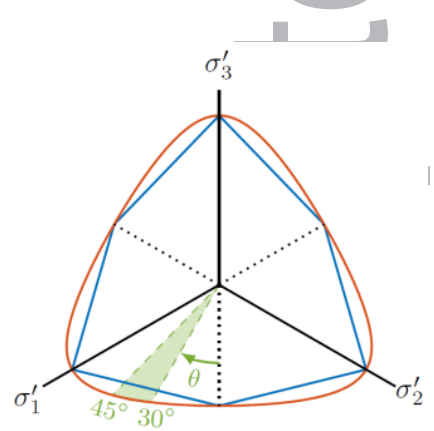

$$
\text { - Mohr-Coulomb }
$$

Bild 15 Grenzbedingungen nach Mohr-Coulomb und Matsuoka-Nakai. Nach [28] liegen Grenzspannungen bei ebenem Verzerrungszustand in einem Bereich für den LodeWinkel von $30^{\circ}<\theta<45^{\circ}$.

Fig. 15 Failure criteria following Mohr-Coulomb and Matsuoka-Nakai. According to [28] limit stresses in a plane distortion state lie in a range for the Lode angle of $30^{\circ}<\theta<45^{\circ}$. 


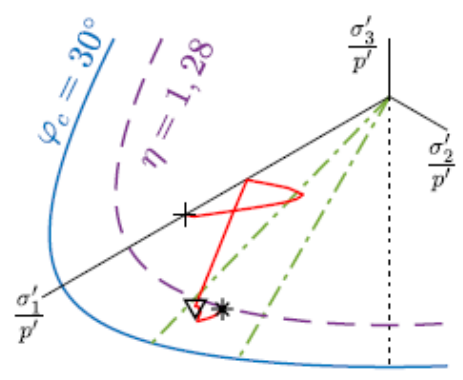

BARO

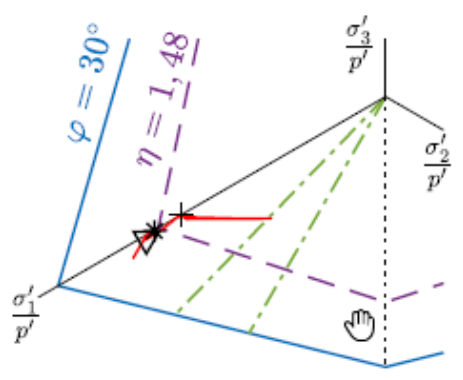

HS

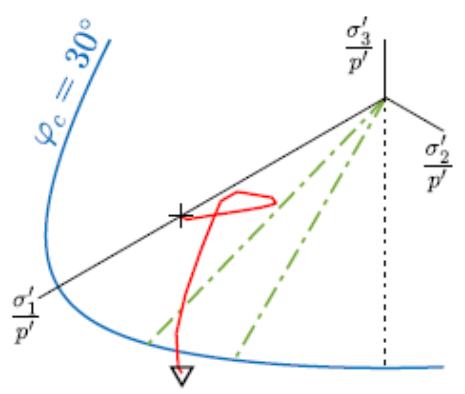

HYPO

Bild 16 Spannungspfade für den in Bild 13 markierten Punkt in einer normierten Deviatorebene mit der jeweiligen Grenzbedingung. Zusätzlich abgebildet ist die Grenzbedingung für die reduzierten Materialparameter sowie der Spannungsbereich für ebene Verzerrungen nach [28]. + Start; $\nabla$ Ende Aushub; * Versagen

Fig. 16 Stress paths for the point marked in Figure 13 in a normalized deviator plane with the respective failure criteria. In addition, the limit condition for the reduced material parameters and the stress range for plane strains according to [28] are shown. + start; $\nabla$ end of excavation; * failure

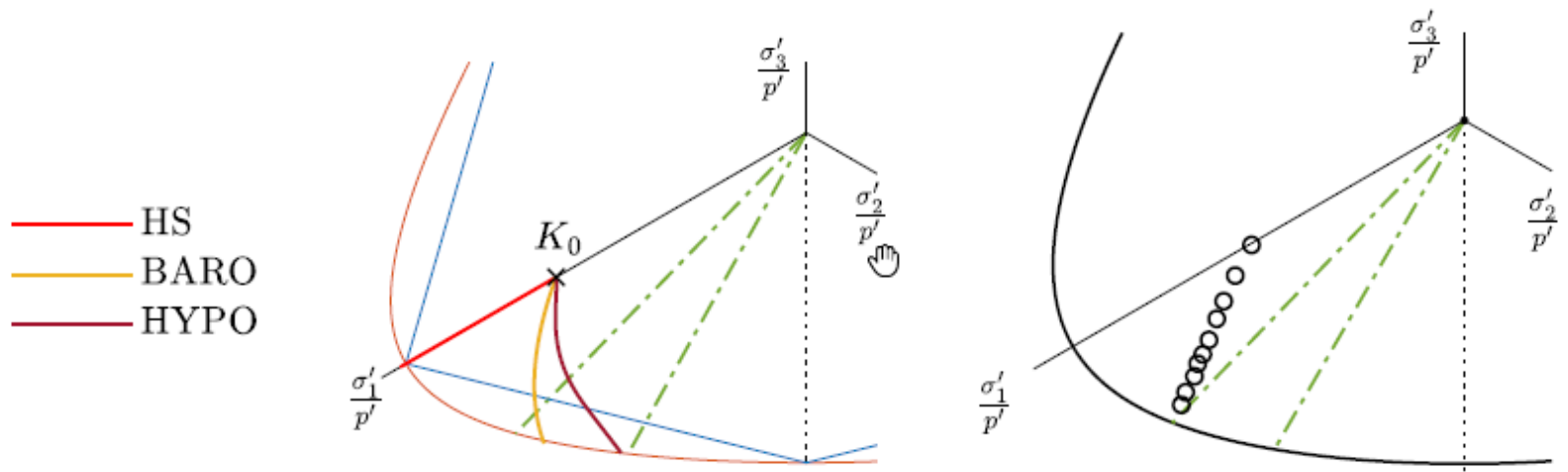

Bild 17 Simulierte $K_{0}$-konsolidierte Biaxialversuche (links), sowie drainierte Laborversuche mit p'=const nach [29] (rechts) mit normalkonsolidiertem Ton

Fig. 17 Simulated $K_{0}$-consolidated biaxial experiments (left), as well as drained laboratory experiments with p'=const after [29] (right) with normal-consolidated clay

Tabelle 1 Verwendete Parameter für HS bei einem Referenzdruck von $\mathrm{p}_{\mathrm{ref}}^{\prime}=100 \mathrm{kPa}$. Parametersatz leicht modifiziert nach [4]

Table 1 Parameters used for HS at a reference pressure of $p_{\text {ref }}^{\prime}=100 \mathrm{kPa}$. Parameter set slightly modified according to [4]

\begin{tabular}{lc|cccccc}
\hline $\begin{array}{l}\varphi^{\prime} \\
\left(^{\circ}\right)\end{array}$ & $\begin{array}{c}\mathrm{c}^{\prime} \\
(\mathrm{kPa})\end{array}$ & $\begin{array}{c}\Psi \\
(-)\end{array}$ & $\begin{array}{c}v_{\mathrm{ur}} \\
(-)\end{array}$ & $\begin{array}{c}\mathrm{E}_{50}^{\mathrm{ref}} \\
(\mathrm{kPa})\end{array}$ & $\begin{array}{c}\mathrm{E}_{\mathrm{oed}}^{\mathrm{ref}} \\
(\mathrm{kPa})\end{array}$ & $\begin{array}{c}\mathrm{E}_{\mathrm{ur}}^{\mathrm{ref}} \\
(\mathrm{kPa})\end{array}$ & $\begin{array}{c}\mathrm{m} \\
(-)\end{array}$ \\
\hline 30 & 0 & 0 & 0,2 & 4100 & 1800 & 14400 & 0,9 \\
\hline \multicolumn{7}{l}{ mod. Param. } & \multicolumn{8}{c}{ original Parameter nach [4] } \\
\hline
\end{tabular}

Tabelle 2 Verwendete Materialparameter für Barodesie [12] und Hypoplastizität [11] mit einer Referenzspannung $\sigma^{*}=1 \mathrm{kPa}$

Table 2 Used parameters for Barodesy [12] and Hypoplasticity [11] at a reference stress $\sigma^{*}=1 \mathrm{kPa}$

\begin{tabular}{ccccc}
\hline$\varphi_{\mathrm{c}}^{\prime}$ & $\mathrm{N}$ & $\lambda^{*}$ & $\kappa^{*}$ & $v$ \\
\hline $30^{\circ}$ & 1 & 0,052 & 0,0075 & 0,2 \\
\hline
\end{tabular}

